\title{
Factors Affecting Recurrence of Idiopathic Granulomatous Mastitis
}

\author{
Halil Ibrahim Tasci ${ }^{1}$, Emin Turk ${ }^{1}$, Ozgur Hilal Erinanc ${ }^{2}$, Serkan Erkan ${ }^{1}$, Ramazan Gundogdu ${ }^{1}$ and Erdal Karagulle ${ }^{1}$ \\ ${ }^{1}$ Department of General Surgery, Baskent University School of Medicine, Ankara, Turkey \\ ${ }^{2}$ Department of Pathology, Baskent University School of Medicine, Ankara, Turkey
}

\begin{abstract}
Objective: To investigate factors that may have an effect on recurrence by retrospectively analysing the data of patients who were followed up and treated for idiopathic granulomatous mastitis in this clinic.

Study Design: Analytic study.

Place and Duration of Study: Department of General Surgery, Faculty of Medicine, Baskent University, Konya and Adana Practice and Research Hospitals between January 2010 and January 2021.

Methodology: The data of patients who were histopathologically diagnosed with granulomatous mastitis were retrospectively analysed. The patients included in the study were divided into two groups: Recurrence (Group 1) and non-recurrence (Group 2). Patients with underlying etiological factors that may cause granulomatous inflammation such as infection, trauma, tuberculosis, sarcoidosis, and autoimmune disease were excluded from the study. The effects of other parameters (involvement area, followup duration, the time from the onset of complaints to diagnosis, side of involvement, breastfeeding, oral contraceptive use, redness, ulceration and/or discharge, preoperative histopathological diagnosis, diagnosis by any of the preoperative imaging techniques, preferred treatment method) on recurrence, were statistically analysed.

Results: Furthermore, the analysis results showed no significant difference between the groups with and without recurrence with regard to any of the other variables such as follow-up period, size of the involvement area, presenting complaints, breast-feeding, preferred treatment alternative (steroid, steroid + surgery, surgery), and preferred surgical technique $(p>0.05)$. The time from the onset of complaints to diagnosis was significantly longer in the recurrence group $(p=0.001)$. In addition, the frequency of oral contraceptive use was statistically significantly higher in the recurrence group (Odds ratio $=7.6, p=0.044$ ).

Conclusion: The results of this study suggest that early diagnosis could prevent recurrence in patients with idiopathic granulomatous mastitis. Prospective randomised controlled studies are needed to support this thought.
\end{abstract}

Key Words: Idiopathic granulomatous mastitis, Malignancy, Oral contraceptive, Steroid.

How to cite this article: Tasci HI, Turk E, Erinanc OH, Erkan S, Gundogdu R, Karagulle E. Factors Affecting Recurrence of Idiopathic Granulomatous Mastitis. J Coll Physicians Surg Pak 2022; 32(02):161-165.

\section{INTRODUCTION}

Granulomatous mastitis is a rare benign chronic inflammatory disease of the breast. ${ }^{1}$ It often mimics inflammatory breast cancer and periductal mastitis clinically and radiologically. ${ }^{2}$ Granulomatous mastitis that occurs without an etiological cause is called idiopathic granulomatous mastitis (IGM). ${ }^{2}$ Clinically, patients present with complaints such as breast mass, pain, and ulceration of the breast skin., Examination findings usually include a firm, painful, palpable mass. ${ }^{5}$

Correspondence to: Dr. Halil Ibrahim Tasci, Department of General Surgery, Baskent University School of

Medicine, Ankara, Turkey

E-mail: okcu6528@gmail.com

Received: August 25, 2021; Revised: November 22, 2021;

Accepted: December 10, 2021

DOI: https://doi.org/10.29271/jcpsp.2022.02.161
There is no standardised approach to the treatment of IGM. ${ }^{6}$ It can frequently recur due to difficulties in diagnosis and the lack of an approach with proven efficacy for treatment. There is also a paucity of literature on this elusive diagnosis precluding a standard course of managementand prediction of outcome.

The aim of this study was to investigate factors that may have an effect on recurrence for IGM.

\section{METHODOLOGY}

This study was approved by the University (Project No. KA21/201). The data of patients who were histopathologically diagnosed with granulomatous mastitis in the Department of General Surgery, between January 2010 and January 2021, were retrospectively analysed. Patients with underlying etiological factors that may cause granulomatous inflammation such as infection, trauma, tuberculosis, sarcoidosis, and autoimmune disease, were excluded.

Breast ultrasound was used as the imaging modality for all 
patients, while magnetic resonance imaging of the breast and/or mammography was used as additional examinations for some patients. Patients with a histopathological diagnosis underwent surgical excision, surgical excision after steroid therapy, or received only steroid therapy.

Patients were evaluated in terms of demographic data, presenting complaints, radiological findings, biopsy method, medical and/or surgical treatments, recurrence, and requirement for reoperation in long-term follow-ups. The patients included in the study were divided into two groups: Recurrence (Group 1) and non-recurrence (Group 2). The effects of other parameters (involvement area, follow-up duration, the time from the onset of complaints to diagnosis, side of involvement, breastfeeding, oral contraceptive use, redness, ulceration and/or discharge, preoperative histopathological diagnosis, diagnosis by any of the preoperative imaging techniques, preferred treatment method) on recurrence were statistically analysed.

SPSS version 21.0 (Statistical Package for the Social Sciences, IBM Corp. Armonk, NY, USA) software package was used for the analyses of this study. The level of significance was set at $p$ $<0.05$ for all analyses. The normality distribution of the data was evaluated by the Kolmogorov-Smirnov test. Categorical variables were presented as frequency tables with percentages, while numerical variables were presented as descriptive measures (mean \pm standard deviation or median (min-max) in non-parametric cases). One-way analysis of variance (ANOVA) was used for inter-group comparisons when parametric conditions were provided, while the Kruskal-Wallis method was used in other cases. The student t-test or Mann-Whitney U-test was used for two-group comparisons. The Chi-square analysis was used to test whether categorical variables were correlated or not, and Pearson or Spearman correlation tests were used to determine the correlation between numerical variables. ROC analysis was performed and cut-off values were calculated for statistically significant numerical variables that may have an effect on predicting recurrence. Univariate and multivariate logistic regression analyses were carried out to identify significantriskfactors for recurrence.

\section{RESULTS}

The mean age of the 63 patients was $34.76 \pm 5.90$ years. The most common presenting complaint of the patients was breast mass (45 patients, $71.4 \%$ ). Of the 63 patients, $23(36.5 \%$ ) had breast pain and 11 (17.5\%) had breast redness. Only 5 (7.9\%) patients had a history of oral contraceptive use. It was learned that 5 (7.9\%) patients did not breastfeed. Twenty-seven (42.9\%) patients had right breast involvement and 36 (57.1\%) patients had left breastinvolvement.

Ultrasound was performed on 63 patients at presentation; and the results were reported as follows: BIRADS 4 lesions in $17(27 \%)$ patients, BIRADS 5 lesions in 2 (3.2\%) patients, dense abscess in 19 (30.2\%), mastitis in 17 (27\%), and an appearance consistent with granulomatous mastitis in only $8(12.7 \%)$ patients. Twenty $(31.7 \%)$ patients underwent magnetic resonance imaging. Of these patients, 1 ( $5 \%$ ) had a dense abscess, $6(30 \%)$ had BIRADS 4 lesions, 1 (5\%) had BIRADS 5 lesions, and 12 (60\%) had granulomatous mastitis. The median size of the breast involvement area was calculated to be $40 \mathrm{~mm}$ (range: $10-100 \mathrm{~mm}$ ) by imaging techniques. Histopathological diagnosis was made after excisional breast biopsy in 13 (20.6\%) patients. Prior to surgical and/or medical treatment, the diagnosis was histopathologically confirmed by fine needle biopsy in 1 (1.6\%) patient and tru-cut biopsy in 49 (77.8\%) patients. The median time from the onset of complaints to diagnosis was one month (range: 1 week-72 months).

Twenty-one (33.3\%) patients underwent no surgical intervention. Twenty-eight (44.4\%) patients underwent wide excision and $2(3.2 \%)$ patients underwent mammoplasty in the same session with mastectomy. Twelve (19\%) patients underwent only abscess drainage. Steroid therapy was not administered to 31 (49.2\%) patients, 15 (23.8\%) patients received preoperative steroid therapy, while $17(27 \%)$ patients received steroid therapy as primary therapy. Three $(4.76 \%)$ patients had leech therapy, one of the traditional medical practices, but they did not benefit from it. A culture study was ordered for 35 (55.5\%) patients, and growth was observed in the cultures of only 4 (11.42\%) patients. After a median follow-up of 15 month (range: 1-126 month), and 24 (38.09\%) patients had a recurrence. The median time to recurrence was 4 month (range: 2-24 month) in these patients.

Table l: Comparative data of group 1 and group 2.

\begin{tabular}{|c|c|c|c|}
\hline & Group $1(n=24)$ & Group $2(n=39)$ & p-value \\
\hline Age (year) & $34.70 \pm 5.76$ & $34.79 \pm 6.06$ & 0.95 \\
\hline $\begin{array}{l}\text { Lateralisation } \\
\text { Right } \\
\text { Left }\end{array}$ & $\begin{array}{l}12(50 \%) \\
12(50 \%)\end{array}$ & $\begin{array}{l}15(38.46 \%) \\
24(61.53 \%)\end{array}$ & 0.36 \\
\hline $\begin{array}{l}\text { OCs use }(n) \\
\text { Yes } \\
\text { No }\end{array}$ & $\begin{array}{l}4(16.6 \%) \\
20(83.3 \%)\end{array}$ & $\begin{array}{l}1(2.56 \%) \\
38(97.43 \%)\end{array}$ & 0.04 \\
\hline $\begin{array}{l}\text { Breastfeeding }(\mathrm{n}) \\
\text { Yes } \\
\text { No }\end{array}$ & $\begin{array}{l}21(87.5 \%) \\
3(12.5 \%)\end{array}$ & $\begin{array}{l}37(94.87 \%) \\
2(5.12 \%)\end{array}$ & 0.29 \\
\hline $\begin{array}{l}\text { Involvement area } \\
(\mathrm{mm})\end{array}$ & $42.5(10-100)$ & $40(10-80)$ & 0.36 \\
\hline $\begin{array}{l}\text { Surgery }(\mathrm{n}) \\
\text { None } \\
\text { Excision } \\
\text { Mastectomy } \\
\text { Drainage }\end{array}$ & $\begin{array}{l}8(33.3 \%) \\
12(50 \%) \\
1(4.16 \%) \\
3(12.5 \%)\end{array}$ & $\begin{array}{l}13(33.3 \%) \\
16(41.02 \%) \\
1(2.56 \%) \\
9(23.07 \%)\end{array}$ & 0.73 \\
\hline $\begin{array}{l}\text { Steroid }(n) \\
\text { None } \\
\text { Preoperative } \\
\text { Primary }\end{array}$ & $\begin{array}{l}12(50 \%) \\
7(29.16 \%) \\
5(20.83 \%)\end{array}$ & $\begin{array}{l}19(48.71 \%) \\
8(20.51 \%) \\
12(30.76 \%)\end{array}$ & 0.60 \\
\hline $\begin{array}{l}\text { Treatment }(n) \\
\text { None } \\
\text { Surgery } \\
\text { Steroid + surgery } \\
\text { Steroid }\end{array}$ & $\begin{array}{l}1(4.16 \%) \\
7(29.16 \%) \\
8(33.3 \%) \\
8(33.3 \%)\end{array}$ & $\begin{array}{l}5(12.82 \%) \\
18(46.15 \%) \\
7(17.94 \%) \\
9(23.07 \%)\end{array}$ & 0.23 \\
\hline $\begin{array}{l}\text { Follow-up } \\
\text { (month) }\end{array}$ & $28(1-126)$ & $9(1-108)$ & 0.44 \\
\hline $\begin{array}{l}\text { Time comp.-dia } \\
\text { (month) }\end{array}$ & $\begin{array}{l}2.5(1 \\
\text { week-24) }\end{array}$ & 1 (1 week-72) & 0.001 \\
\hline
\end{tabular}

The comparative data of Group 1 and Group 2 are summarised in Table I. Accordingly, although Group 1 had a larger breast 
involvement area [42.5 (range, 10-100) mm in Group 1, 40 (range, 10-80) mm in Group 2], there was no statistically significant difference between the groups $(p=0.36)$. There was no effect of the follow-up duration on recurrence $(p=0.44)$. The time from the onset of complaints to diagnosis has been considered an important parameter that gave an idea about early diagnosis or delayed diagnosis. Statistically, the time from the onset of complaints to diagnosis was significantly longer in the recurrence group $(p=0.001)$. Apart from these, there was no significant difference between the groups with regards to any of the factors, such as right or left breast involvement $(p=0.36)$, breastfeeding $(p=0.29)$, breast redness $(p=0.21)$, ulceration and/or discharge $(p=0.41)$, preoperative histopathological diagnosis $(p=0.52)$, diagnosis by any of the preoperative imaging techniques $(p=0.38)$, preferred surgical technique $(p=0.73)$, steroid therapy regimen $(p=0.60)$, or preferred treatment method (surgery, steroid + surgery, steroid) $(p=0.23)$. According to the results of the Chi-square test, the frequency of oral contraceptive use was statistically significantly higher in the recurrence group and the odds value was calculated as 7.6 in the risk analysis $(p=0.044)$. The correlation analyses revealed a negative correlation between breastfeeding and the size of the mass detected by imaging techniques ( $p=0.007)$, a positive correlation between recurrence and the time from the onset of complaints to diagnosis $(p<0.001)$ and between recurrence and use of oral contraceptives $(p=0.04)$. The two-way ANOVA showed that oral contraceptive use and breastfeeding were separately and together effective in recurrence time $(p<0.05)$.

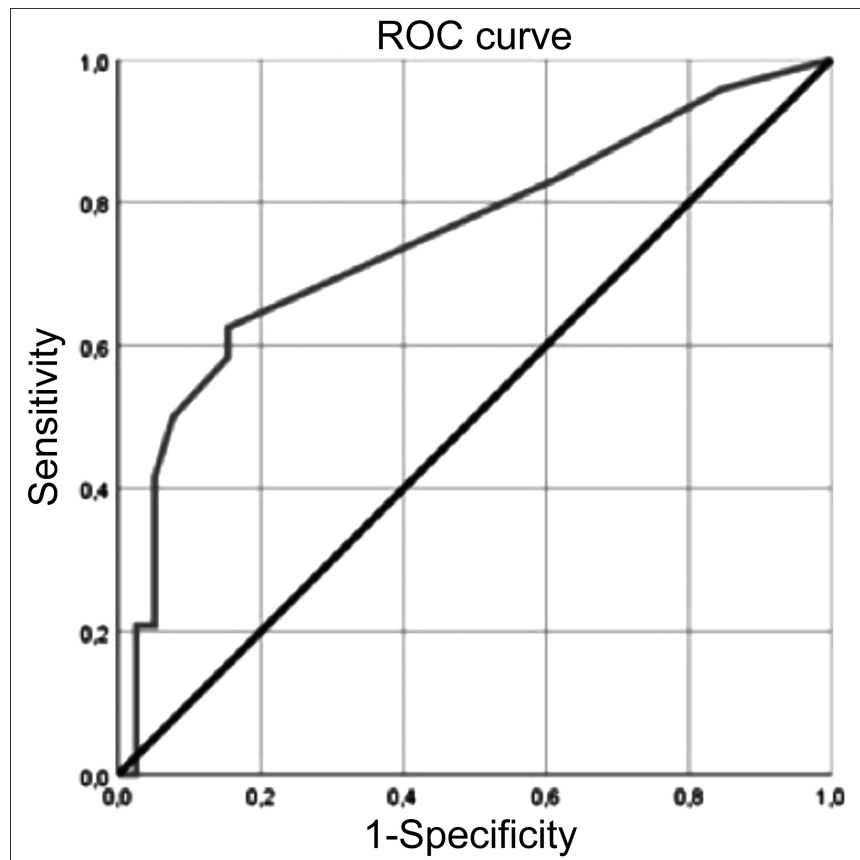

Figure 1:The roc (receiver operating characteristic) curve of the time from the onset of complaints to diagnosis.

The ROC (receiver operating characteristic) analysis showed that only the time from the onset of complaints to diagnosis was statistically significant with a cut-off value of 2.5 months $(p=0.01$, Figure 1$)$. According to the risk analysis using this value, a time from the onset of complaints to diagnosis longer than 2.5 months significantly increased the risk of recurrence (odds ratio $=12, p<0.001$ ). Univariate and multivariate logistic regression analysis was performed that was carried out to identify the significant factors that may be effective in determining recurrence. In this analysis, the variables of oral contraceptive use, the presence of breast redness, the time from the onset of complaints to diagnosis, and the size detected by imaging were examined. As a result, only the time from the onset of complaints to diagnosis was found to be effective in determining recurrence $(p=0.001$, Table II).

Table II: Multivariate logistic regression analysis of significant factors that may be effective in determining recurrence.

\begin{tabular}{|c|c|c|c|c|}
\hline \multirow{2}{*}{ Rısk factor } & \multirow{2}{*}{$\begin{array}{l}\text { Odds } \\
\text { Ratio }\end{array}$} & \multicolumn{2}{|c|}{$95 \% \mathrm{Cl}$} & \multirow{2}{*}{ p-value } \\
\hline & & lower & upper & \\
\hline $\begin{array}{l}\text { OCs use } \\
\text { Breast redness } \\
\text { Time comp. - } \\
\text { dia. }(<2,5 / \geq 2,5) \\
\text { İnvolvement area }(\mathrm{mm})\end{array}$ & $\begin{array}{l}8.60 \\
1.48 \\
11.98 \\
1.01\end{array}$ & $\begin{array}{l}0.71 \\
0.29 \\
2.60 \\
0.98\end{array}$ & $\begin{array}{l}104.25 \\
7.18 \\
55.13 \\
1.04\end{array}$ & $\begin{array}{l}0.09 \\
0.65 \\
0.001 \\
0.26\end{array}$ \\
\hline
\end{tabular}

\section{DISCUSSION}

IGM refers to a non-specific chronic inflammatory disease of the breast of an unknown etiology accompanied by granulation. ${ }^{7}$ It is a rare pathology predominantly seen in Asian and Hispanic races. Although there are studies reporting an increased risk of IGM with the use of oral contraceptives, infection, pregnancy, breastfeeding, breast trauma, hyperprolactinemia, antitrypsin, and antidepressants, the exact etiological cause is uncertain. ${ }^{8}$ Of the patients included in this study, $5(7.9 \%)$ had a history of oral contraceptive use and $58(92.1 \%)$ had a history of breastfeeding. IGM can be seen at any age, but it primarily occurs in women of childbearing age. ${ }^{9}$ Its incidence has been reported as 2.4 cases per 100,000 people in the USA, with an average age of 34 years. ${ }^{10}$ All of the patients included in this study were females, with a mean age of $34.76 \pm 5.90$ years. In the study, it was found that age had no effect on recurrence.

Patients usually present with the complaint of a rapidly growing breast mass. The complaint of mass may be accompanied by inflammation, abscess, nipple retraction, and fistula with chronic discharge.$^{11}$ It equally affects the right or left breast and all four quadrants of the breast. ${ }^{12}$ The most common presenting complaint of patients included in this study was breast mass (45 patients, $69.7 \%$ ). While there was no bilateral breast involvement, 27 (42.9\%) patients had right breast involvement and 36 (57.1\%) patients had left breast involvement. Considering the side and complaint variables in this study, it was seen that both variables had no effecton recurrence.

Radiological techniques are not diagnostic for IGM. On magnetic resonance imaging, it is seen as focally homogeneous growing masses with irregular borders. ${ }^{13}$ Moreover, skin thick- 
ening, parenchymal edema, and asymmetrical enhancement can be seen. ${ }^{14}$ All of patients underwent breast ultrasound, which showed an appearance consistent with granulomatous mastitis in only $8(12.7 \%)$ patients. In addition, $20(31.7 \%)$ patients underwent magnetic resonance imaging and 12 (60\%) of them were diagnosed with granulomatous mastitis. Only 4 $(6.3 \%)$ of the patients underwent mammography, which showed a non-specific asymmetric density increase in all of them. According to the data of this study, the choice of magnetic resonance imaging as an imaging technique for patients with suspected granulomatous mastitis can prevent delays in diagnosis and therefore in treatment.

Both physical examination findings and imagings, usually fail to differentiate between IGM and malignancy. A definitive diagnosis is required to determine the treatment strategy. At this point, histopathological examination is essential to prevent delays in diagnosis and treatment. Of patients in this study, 1 (1.6\%) was histopathologically diagnosed before treatment by fine-needle biopsy and 49 (77.8\%) by tru-cut biopsy.

There are many different approaches to the treatment of IGM, whereas there is no standardised treatment protocol yet. The main choice of the treatment methods includes surgery, steroids, and rarely immunosuppressive agents. These can be used separately or in various combinations. ${ }^{15}$ There are publications suggesting that surgical treatment is more effective than steroid treatment; however, there are also papers stating that surgery to be performed after steroid therapy, is more effective in terms of both cosmetic results and recurrence. ${ }^{11,14}$ On the other hand, studies have shown more negative outcomes of primary steroid therapy administered with a conservative approach compared to other treatment methods in terms of both recurrence and cosmetic results. ${ }^{14}$ Besides these treatment approaches, postoperative steroid therapy (especially in patients unresponsive to surgery), regional steroid injection, hydroxychloroquine, methotrexate, azathioprine, and colchicine have also been used for the treatment. ${ }^{8}$

Due to the difficulties in treatment and the frequent incidence of recurrence, it is possible to encounter patients, who resort to traditional medical practices both in our country and in the world, and academic studies on this subject. ${ }^{16}$ Three $(4.76 \%)$ of our patients had leech therapy, but they did not benefit from it. These patients underwent surgical excision after 8 weeks of steroid therapy.

In this study, the time from the onset of complaints to diagnosis has been considered an important parameter that gives an idea about early diagnosis or delayed diagnosis. Statistically, the time from the onset of complaints to diagnosis was significantly longer in the recurrence group $(p=0.001)$. Apart from these, there was no significant difference between the groups with regard to any of the factors, such as right or left breast involvement $(p=0.36)$, breastfeeding $(p=0.29)$, breast redness $(p=0.21)$, ulceration and/or discharge $(p=0.41)$, preoperative histopathological diagnosis $(p=0.52)$, diagnosis by any of the preoperative imaging techniques $(p=0.38)$, preferred surgical technique $(p=0.73)$, steroid therapy regimen $(p=0.60)$, or preferred treatment method (surgery, steroid + surgery, steroid, $p=0.23$ ).

The limitations of this study are: collecting the data from patients' records and operative reports and the absence of objective examination findings due to the retrospective nature of the study.

\section{CONCLUSION}

This study aiming to analyse factors affecting recurrences, demonstrated that demographic, clinical data, radiological findings, and treatment methods of the patients statistically had no effect on recurrence. Only the time from the onset of complaints to diagnosis, which may be an indicator of delay in diagnosis and treatment, and the use of oral contraceptives were found to have an effect on recurrence. The results of this study suggest that early diagnosis could prevent recurrence in patients with idiopathic granulomatous mastitis. Prospective randomised controlled studies are needed to support this thought.

\section{FUNDING:}

This study was supported by Baskent University Research Fund.

\section{ETHICALAPPROVAL:}

Ethical approval of this study was obtained from Medical and Health Sciences Research Board of Baskent University (Project No. KA21/201), prior to initiation of the research work.

\section{PATIENTS' CONSENT:}

Informed consents were obtained from all patients to publish the data concerning this study.

\section{CONFLICT OF INTEREST:}

The authors declared no conflict of interest.

\section{AUTHORS' CONTRIBUTION:}

HIT: Conception of work, data collection and interpretation, literature search and manuscript writing, final approval of the version to be published.

ET: Conception of work, design, final approval of the version to be published.

OHE: Data collection, conception of work, final approval of the version to be published.

SE, RG, EK: Conception of work, final approval of the version to be published.

All authors agreed to be accountable for all aspects of the work in ensuring that questions related to the accuracy or integrity of any part of the work are appropriately investigated and resolved.

\section{REFERENCES}

1. Montazer M, Dadashzadeh M, Moosavi Toomatari SE. Comparison of the outcome of low dose and high-dose corticosteroid in the treatment of Idiopathic granulomatous mastitis. Asian Pac J Cancer Prev 2020; 21(4):993-6. doi: 10.31557/APJCP.2020.21.4.993.

2. Chalmers R, McClellan P, Silva V, Shutt N, Restini C. Red 
flags for the differential diagnosis of granulomatous mastitis: A case report. J Med Case Rep 2020; 14(1):215. Doi: 10.1186/s13256-020-02563-x.

3. Perera R, Ratnasooriya C, Perera N, Fernando N, Senevirathne B. Efficacy of surgical treatment in the management of idiopathic granulomatous mastitis: an institutional experience in Sri Lanka. Sri Lanka J Surg 2020; 38(3): 21-4. Doi: 10.4038/sljs.v38i3.8760.

4. Zhang X, Li Y, Zhou Y, Liu D, Chen L, Niu K, et al. A systematic surgical approach for the treatment of idiopathic granulomatous mastitis: A case series. Gland Surg 2020; 9(2): 261-70. Doi: 10.21037/gs.2020.02.06.

5. Shabnam N, Choudhury IM, Ahsan T. Treatment modality of Idiopathic granulomatous mastitis with surgery alone or in combination with steroid. Bioresearch Communications 2021; 1:924-6.

6. Akcan A, Akyıldız H, Deneme MA, Akgun H, Arıtas Y. Granulomatous lobular mastitis: A complex diagnostic and therapeutic problem. World J Surg 2006; 30(8):1403-9. Doi: 10.1007/s00268-005-0476-0.

7. Khalaf AM, Al-Shimy GG, Hefny AAH. The myth of Idiopathic granulomatous mastitis. AIMJ 2020; 1(4):116-23. Doi: 10.21608/aimj.2020.46076.1335.

8. Gemici E, Akdoğan Gemici A. Effectiveness of local methylprednisolone application in the treatment of Idiopathic granulomatosis mastitis. IKSSTD 2020; 12(3):254-7.

9. Chandanwale S, Naragude P, Shetty A, Sawadkar M, Raj A, Bhide $A$, et al. Cytomorphological spectrum of granulomatous mastitis: A study of 33 cases. Eur J Breast Health 2020; 16(2):146-51. Doi: 10.5152/ejbh.2020.5185.
10. Berganza PF, Ramos MS. Granulomatous mastitis, radiohistological correlation. Open J Radiol 2020; 10(04):223-9 Doi: 10.4236/ojrad.2020.104022.

11. Yabanoğlu H, Çolakoğlu T, Belli S, Aytac HO, Bolat FA, Pourbagher $A$, et al. A comparative study of conservative versus surgical treatment protocols for 77 patients with Idiopathic granulomatous mastitis. Breast J 2015; 21(4):363-9. Doi: 10.1111/tbj.12415.

12. Azlina AF, Ariza Z, Arni T, Hisham AN. Chronic granulomatous mastitis: diagnostic and therapeutic considerations. World J Surg 2003; 27(5):515-8. doi: 10.1007/s00268-003-6806-1.

13. Yip $\mathrm{CH}$, Jayaram $\mathrm{G}$, Swain $M$. The value of cytology in granulomatous mastitis: A report of 16 cases from Malaysia. Aust N Z J Surg 2000; 70(2):103-5. doi: 10.1046/j.1440-1622.2000.01764.x.

14. Gündeş E, Yıldırım MA, Küçükkartallar T, Aksoy F, Tekin A, Çakir $\mathrm{M}$, et al.Combined treatment method comparison of medical and surgical treatment in Idiopathic granulomatous mastitis. Int Med Sci Clin Inven 2018; 5(4):3737-42. Doi: 10.18535/ijmsci/v5i4.05.

15. Conte AB, Nyingone S, Jayi S, Soule HM, Alaoui FZF, Chaara $\mathrm{H}$, et al. Therapeutic and diagnostic challenge of Idiopathic granulomatous mastitis: A case report and review of the literature. J Women's Health Dev 2020; 3(3):185-93.Doi: 10.26502/fjwhd.2644-28840030.

16. Zhang $\mathrm{X}, \mathrm{Li} \mathrm{J}, \mathrm{Hu} \mathrm{XJ}$. Postoperative Yanghe decoction regimen improves outcomes for idiopathic granulomatous mastitis: A retrospective cohort study. Medicine (Baltimore). 2020; 99(45):e23136. Doi: 10.1097/MD. 0000000000023136 